\title{
Simulation Tools for Detector Performance and Calibration at European XFEL
}

\author{
Ashley Joy, Markus Bohlen, Steffen Hauf, Burkhard C. Heisen, Andreas Koch, Markus Kuster, \\ Jolanta Sztuk-Dambietz, Monica Turcato, Matthew Wing and Christopher Youngman
}

\begin{abstract}
The detectors to be used at the European XFEL have to deal with the unique time structure of the machine, delivering up to 2700 pulses, with a repetition rate of $4.5 \mathrm{MHz}$, ten times per second, the very high photon flux of up to $10^{12}$ photons/pulse and the need to combine single-photon sensitivity and a large dynamic range. These machine properties present a challenge for the large-area $2 \mathrm{D}$ imaging detectors to be used at European XFEL. In order to thoroughly characterize the detectors, optimize their performance and the required calibration concepts, as well as give estimates of the expected scientific performance in a wide range of experimental scenarios, we are currently pursuing different simulation projects.
\end{abstract}

\section{INTRODUCTION}

$\mathbf{T}$ HE European X-ray Free Electron Laser (XFEL) distinguishes itself from existing facilites like the SLAC Linac Coherent Light Source (LCLS), USA and the SPring8 Angstrom Compact free electron LAser (SACLA), Japan, by its high repetition rate and high peak brilliance. The machine will deliver up to 27000 pulses/s amounting to $5 \times 10^{33}$ photons $/ \mathrm{s} / \mathrm{mm}^{2} / \operatorname{mrad}^{2}(0.1 \%$ bandwidth, energy range: $0.25 \mathrm{keV}-25 \mathrm{keV}$ ) at $4.5 \mathrm{MHz}$ repetition rate. This poses unique challenges in terms of performance and radiation hardness for the 2D X-ray detectors which are to be used at the instrumental stations.

In order to thoroughly characterize these detectors, optimize their performance and the required calibration concepts, as well as give estimates of the expected scientific performance in a wide range of experimental scenarios, we are currently pursuing different simulation projects.

Initially, small self-contained simulation tools were developed, e.g. for estimating the performance of calibration sources or for estimates on the performance of the large 2D pixel detectors; the results of which are presented here.

Additionally, we are currently developing a large-scale simulation toolkit, which will be integrated into the European XFEL software framework, Karabo [1]. It is envisaged that this framework will be available to XFEL facility users and allow the simulation of the 2D pixel detectors used at European XFEL - starting from the photons incident on the detector down to the level of the read-out electronics.

Manuscript received May 9, 2014

A. Joy and M. Wing are at University College London, London, U.K (email: a.joy@ucl.ac.uk).

M. Bohlen, S. Hauf, B.C. Heisen, A. Koch, M. Kuster, J. Sztuk-Dambietz, M. Turcato and C. Youngman are with European X-ray Free Electron Laser Facility GmbH, Hamburg, Germany (e-mail: steffen.hauf@xfel.eu).

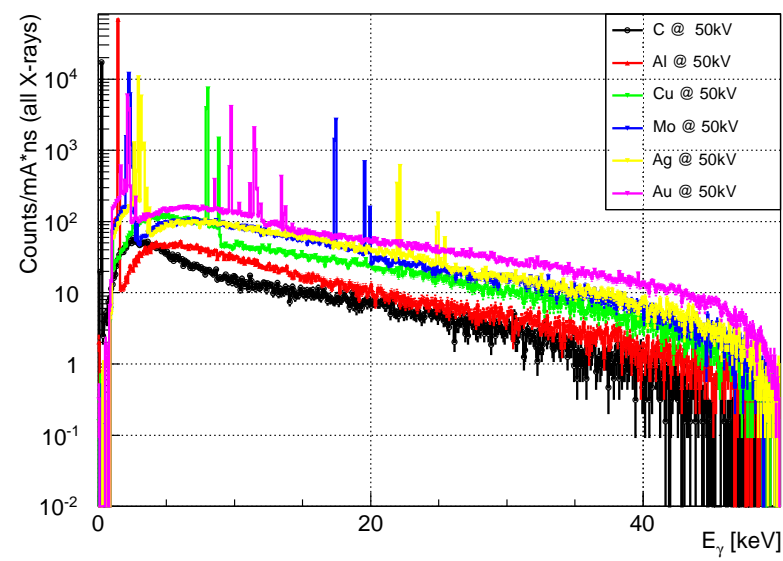

Fig. 1: Geant4 simulation of the X-ray spectra generated by an electron gun based X-ray source using different target materials: $\mathrm{C}, \mathrm{Al}, \mathrm{Cu}, \mathrm{Mo}, \mathrm{Ag}$ and $\mathrm{Au}$. Each simulation consisted of 40 Million primary electrons at $50 \mathrm{keV}$. The target angle was set to $18^{\circ}$ at which $\mathrm{X}$-ray production was found to be most efficient.

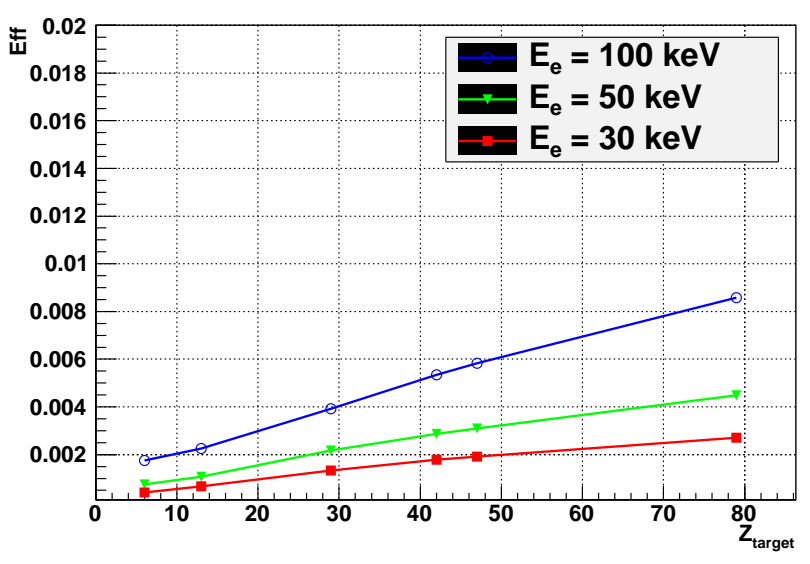

Fig. 2: Conversion efficiency of incident primary electrons into Bremsstrahlung X-rays depending on target material. Estimates for incident electrons at energies of $30 \mathrm{keV}, 50 \mathrm{keV}$ and $100 \mathrm{keV}$ and elements $\mathrm{C}(\mathrm{Z}=6), \mathrm{Al}(\mathrm{Z}=13), \mathrm{Cu}(\mathrm{Z}=29), \mathrm{Mo}$ $(Z=42), A g(Z=47)$ and $\mathrm{Au}(Z=79)$ are shown. Each simulation consisted of 40 Million primary electrons. The target angle was set to $18^{\circ}$ at which X-ray production was found to be most efficient.

\section{X-Ray Calibration Source Simulations}

Calibration of the 2D Mega-pixel detectors with $4.5 \mathrm{MHz}$ readout rate will pose unique challenges for the laboratory 


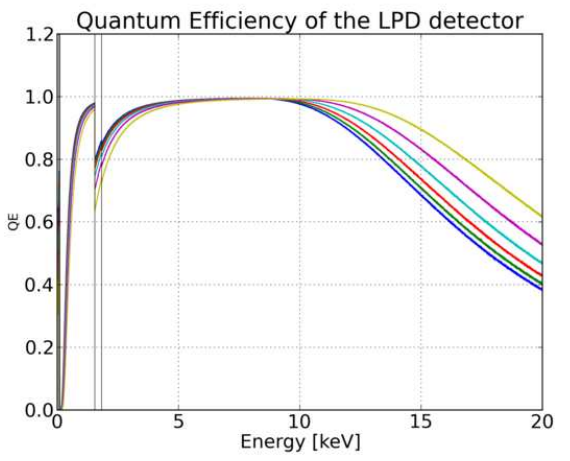

(a)

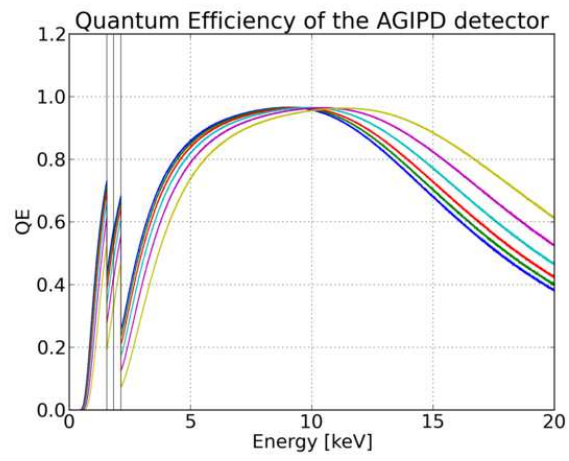

(b)

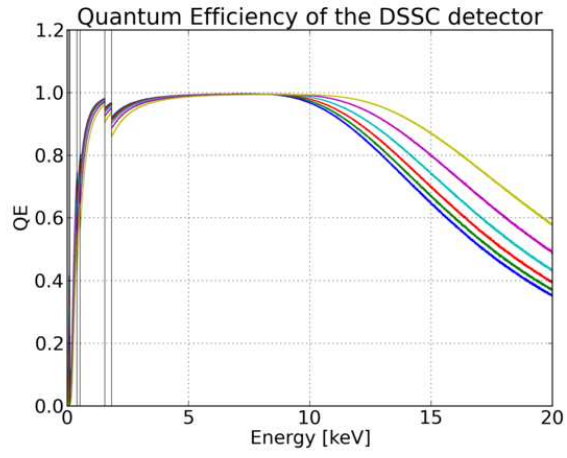

(c)

Fig. 3: Geant4-based quantum efficiency simulations for the three large 2D pixel detectors with $\mathrm{MHz}$ readout capability at European XFEL. The individual absorption edges, determined by the respective detectors' entrance windows, are visible. For each detector the efficiency was simulated at different incidence angles (from lower-most curve at the high-energy end of the plots to top-most curve): $0^{\circ}, 20^{\circ}, 30^{\circ}, 40^{\circ}, 50^{\circ}$ and $60^{\circ}$.

infrastructure at European XFEL. In order to allow for a realistic representation of XFEL operating conditions, calibration measurements must be performed with a time structure and dynamic ranges similar to the FEL.

Therefore, the laboratory calibration sources must provide pulses of up to $10^{3}$ photons per pixel and pulse. As part of a continuous optimization strategy for this infrastructure a Geant4-based Monte Carlo simulation [2], [3] (Geant4 9.4.p03) has been created. It currently allows for performance estimates of different source geometries for an electron beam incident on different target materials. The Penelope physics package [5] is used, as it was found to be better suited for this specific simulation task than Livermore-based physics [5] simulations [6]. Fig. 1 shows a estimate of the X-ray spectrum (fluorescence and Bremsstrahlung) generated by different target materials. A estimate of conversion efficiency for electrons to Bremsstrahlung X-rays, depending on incident electron energy, is shown in Fig. 2. A more detailed discussion of the results from this simulation tool can be found in [4].

In parallel the large 2D detectors, which are to be used at European XFEL - AGIPD, LPD and DSSC - have been modeled in Geant4 9.6 using Livermore-based physics. These models allow follow-up in-house performance estimates in addition to the simulation results provided by the detector consortia [7]-[11]. The models can be integrated with the Xray source simulation and optionally include the influence of the diverse environmental conditions of the instrument stations. Fig. 3 shows quantum efficiency plots for the aforementioned 2D detectors with $\mathrm{MHz}$ readout capabilities. Additional simulations verified that the energy-dependent variation of efficiency is due to back scattering (reduction for large angles at small energies) and geometric length (enhancement for large angles at high energies). It is apparent from the figure that all three detectors will experience an vignetting effect at larger incidence angles and energies above $10 \mathrm{keV}$.

\section{X-CSIT: The X-Ray Camera Simulation ToOlKit}

The X-ray Camera SImulation Toolkit (X-CSIT) is a modular toolkit for the simulation of semi-conductor pixel detectors. It will simulate a semi-conductor pixel detector from the incident photon down to the characteristics of the read-out electronics. The toolkit is designed to account for the wide variations in designs of semi-conductor detectors and can be used to build a simulation for a specific detector.

At XFEL, X-CSIT will be used to create simulations of the three bespoke detectors being built for the facility. These simulations will then be made available to users via the integration of X-CSIT into Karabo.

$\mathrm{X}$-CSIT is split into three sub-simulations in a chain, with each simulation giving an output that the next simulation uses as an input. The three simulations are a particle simulation, a charge simulation and an electronics simulation. These simulations cover, respectively: the incoming photons and their interaction with the semi-conductor material, the motion of charge created by the photon interactions within the semiconductor material, and the electronic response of the detector to charge deposited within a pixel.

The particle simulation uses Geant 4 version 9.6 to simulate photon and electron interactions down to approximately the low energy limit of the Livermore-based physics list of $250 \mathrm{eV}$. The charge simulation and electronics simulations will be written specifically for X-CSIT.

Currently a first prototype of X-CSIT has been implemented: a simple Geant 4 simulation has been integrated into the framework as the particle simulation. Notably, the simulation accepts input and provides output in the framework formats.

\section{A. Design}

$\mathrm{X}$-CSIT is designed to be modular and by design separates out the major components of the simulation. This allows for easy modification, upgrade and validation of individual aspects of the simulation. This feature also allows each stage of the simulation to be run separately, as is expected to be done when 


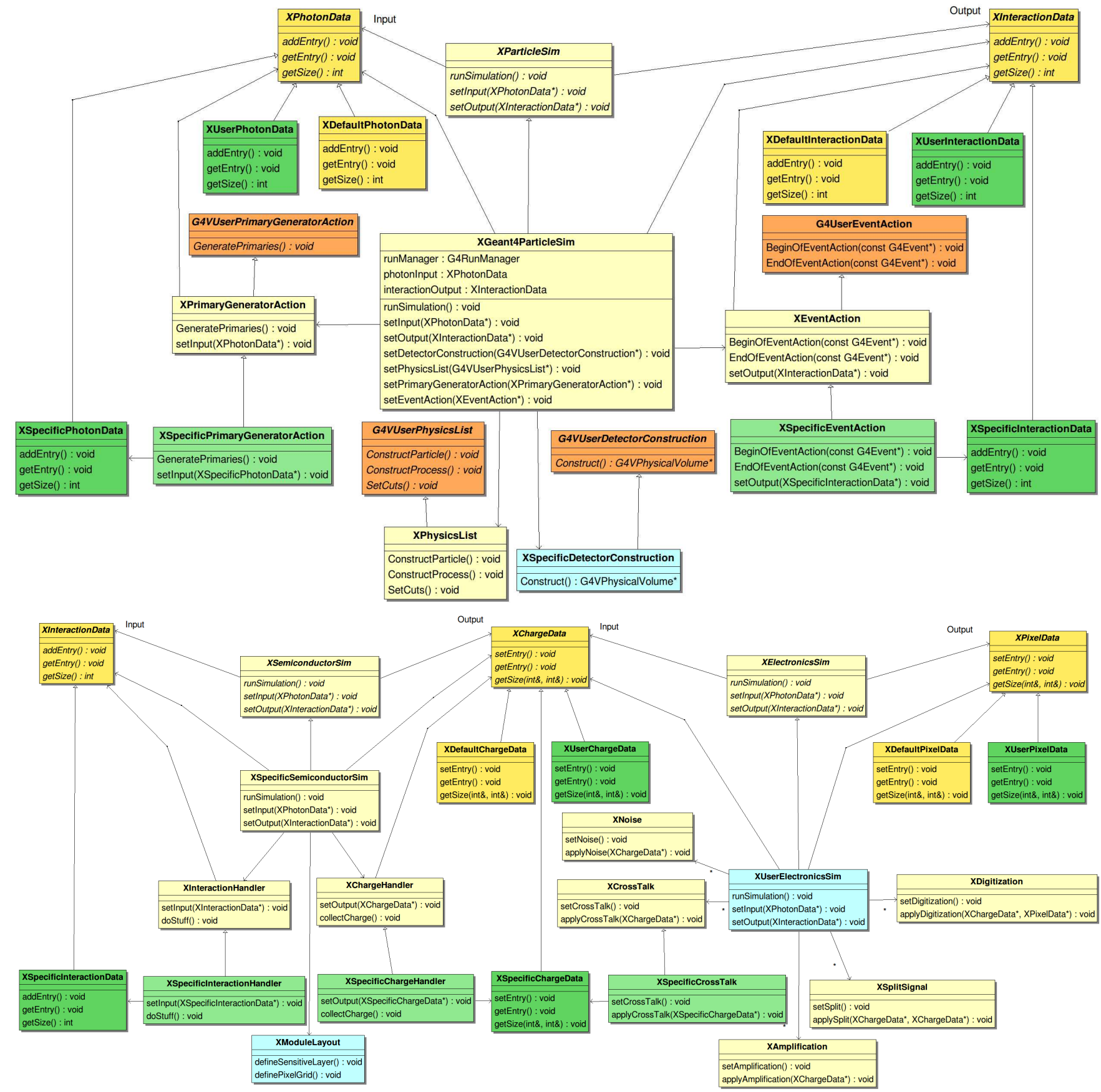

Fig. 4: A UML diagram showing the current class design of X-CSIT. The diagram is split such that the right edge of the top half leads into the left edge of the bottom half. Shown in yellow are the provided classes, red are classes from Geant 4 , blue are classes the user must provide and green are examples of optional classes the user could add to tailor their simulation. Data container classes are shown in a darker shade. The top row of classes are the abstract class layer from which the other classes inherit. The top half shows the particle simulation classes as currently implimented, the bottom half shows the classes for the charge and electronics simulations as they are planned to be implemented. The interfaces of the adder, setter and getter functions are specific to the data class but are not shown for brevity.

$\mathrm{X}$-CSIT is integrated into Karabo, and be run at varying levels of concurrency.

Each of the three simulation components is based on a single abstract base class which the concrete implementation of a simulation will inherit from. These abstract base classes each define an input and output of the simulation compatible with the framework's data classes. The data classes likewise define abstract interfaces with setter and getter functions for the minimum of data required to be stored. How the data is stored internally is left to the concrete implementation. XCSIT will provide default data classes though: only if the user chooses to store or access data differently, will they have to 
provide an alternative implementation. Examples of where this could be done are shown in green in the UML diagram in Fig. 4.

The four data types defined are:

- the photon input to the particle simulation,

- the generated charge and location of this charge as output by the particle simulation,

- the collected charge output by the charge simulation,

- the digital signal output of the electronics simulation.

Each output serves as the input for the subsequent simulation stage.

The Geant 4 simulation will be entirely contained within the particle simulation class to maintain the modular nature of $\mathrm{X}$ CSIT. Interface classes will be provided for the Geant4 component, such that the user only needs to provide a definition of their detector's geometry.

The charge simulation will take the charge deposited in the semi-conductor and calculate where it is deposited on the pixel grid. Features include charge spreading over multiple pixels and plasma effects in areas of high charge concentration. The simulation is capable of simulating non-regular pixel geometries as is the case for the DSSC which uses hexagonal pixels.

The electronics simulation will be the most flexible part of the toolkit. Because semi-conductor detectors vary heavily in how their electronics work, the electronics simulation will provide a wide variety of functions which can simulate the effect of various components, such as functions for amplification, digitization, splitting a signal and various kinds of electronic noise. The user can then define in what order the components are placed together and the degree of effect they have on the signal. For the integration into Karabo it is envisaged that each electronic simulation component will be contained within a Karabo device, with the data classes using Karabo-compatible storage to allow it to handle intersimulation communication. This will allow users to build the electronics simulation visually, using the Karabo GUI to place and link the electronics simulation components.

While X-CSIT is intended to be open for users to modify, it is also designed to help minimise the amount of work needed to create a simulation of a detector and will include as many general-use classes as possible, particularly in the Geant 4 and electronics simulation.

\section{B. Current Status}

Thus far, the framework for X-CSIT, including all abstract classes and several example data classes, has been written and has undergone unit testing. In addition, Geant 4 has been integrated into the particle simulation of the framework, including the necessary code to fire photons at a detector defined by the input data class and output charge interaction data back to the framework.

For testing, a Geant4 simulation consisting of a single block of silicon, $500 \mu \mathrm{m}$ thick with a $1 \mu \mathrm{m}$ thick entrance window, has been implemented. All photons enter the silicon at the normal and the block is sufficiently large that scattering through edges is negligible. As a further proof of concept the
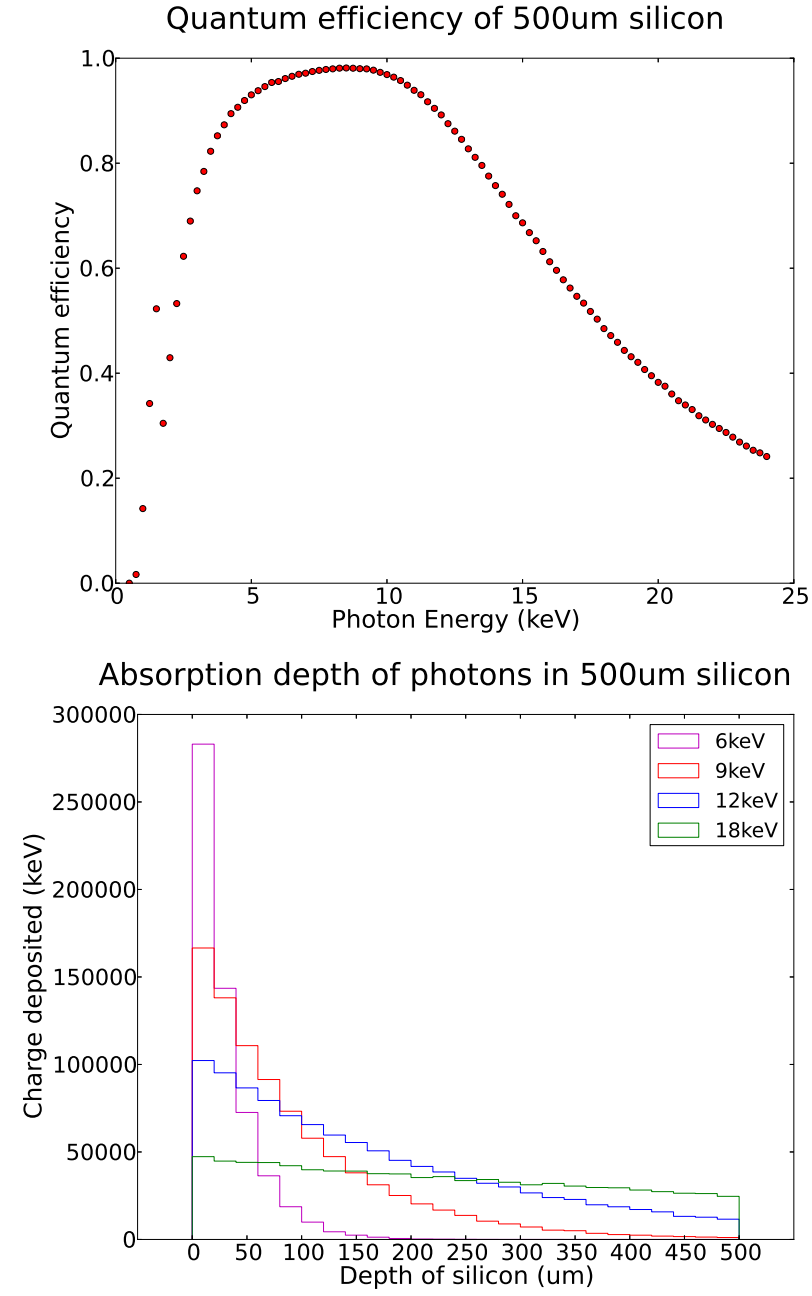

Fig. 5: An X-CSIT driven Geant4 simulation of the quantum efficiency (top panel) and absorption depth (bottom panel) for a $500 \mu \mathrm{m}$ thick silicon detector with a $1 \mu \mathrm{m}$ thick aluminum entrance window. The simulation consisted of 100,000 primary photons for each energy value.

framework has been linked to the python interpreter and can pass data to it live. This was done to demonstrate that $\mathrm{X}$ CSIT can integrate with matplotlib [12], a python plotting library, which will be used for all plotting within Karabo. The plots in Fig. 5 were made by X-CSIT passing data directly to matplotlib.

Fig. 5 shows preliminary results of quantum-efficiency and absorption-depth simulations from this first implementation. The quantum efficiency plot shows the declining absorption rate above $10 \mathrm{keV}$ as the silicon becomes more translucent at higher energies, while the lower energies show the absorption edges of aluminium and silicon. The absorption depth graph shows the characteristic exponential decay in photon absorption according to the Beer-Lambert law. The plots show the expected behaviour and demonstrate that the simulation and framework are qualitatively working. A quantitative analysis of simulation accuracy will be reported at a later time as part of the planned validation work. 


\section{Summary AND PRospects}

The self-contained X-ray generator and detector simulation tools presented in Section II are already actively being used by members of the detector development group at European XFEL.

$\mathrm{X}$-CSIT is still in early development and prototyping. Features still to be added include: a charge carrier simulation including charge spreading and plasma effects, an electronics simulation toolkit, an expanded Geant 4 simulation that handles detector modules and better integration of plotting libraries for visualisation and analysis.

As part of its use at XFEL, simulations of LPD, AGIPD and DSSC will be written using X-CSIT and integrated into Karabo. These simulations are planned to be made available to XFEL users and written so that they can replace detectors in the data chain, allowing users to test certain aspects of their analysis before taking data.

Importantly, there are plans to validate X-CSIT using data taken by the prototype detectors available at XFEL.

\section{REFERENCES}

[1] B.C. Heisen, et al., "Karabo: an integrated software framework combining control, data management and scientific computing tasks", ICALEPS Proceedings, San Francisco, U.S.A., 2013.

[2] S. Agostinelli, et al., "Geant4 a simulation toolkit", Nucl. Instr. Meth A, 506, 250-303, 2003.

[3] J. Allison, et al., "Geant4 developments and applications", IEEE Trans. Nucl. Sci., 53, 270-278, 2006.

[4] J. Sztuk-Dambietz, et al., "Status of detector development for the European XFEL.", SPIE Optics+Optoelectronics. International Society for Optics and Photonics, 2013.

[5] CERN, "Geant4 Phyics Reference Manual 9.6", http://geant4.web.cern.ch/geant4/UserDocumentation/UsersGuides/ PhysicsReferenceManualffo/PhysicsReferenceManual.pdf, Dec, 2013.

[6] M. Guthoff, et al., "Geant4 Simulation of a filtered X-ray source for radiation damage studies", doi: arXiv:1205.1973, 2012.

[7] J. Becker, C. Gutt, H. Graafsma, "Simulation study of the impact of AGIPD design choices on X-ray Photon Correlation Spectroscopy utilizing the intensity autocorrelation technique", J. Inst, 6, P11005, 2011.

[8] J. Becker, D. Pennicard, H. Graafsma, "The detector simulation toolkit HORUS", J. Inst, 7, C10009, 2012.

[9] A. Koch, et al., "Performance of an LPD prototype at MHz frame rate under FEL and Synchrotron radiation", IWORID Proceedings, Paris, France, 2013.

[10] M. Porro, et al., "Expected performance of the DEPFET sensor with signal compression: A large format X-ray imager with mega-frame readout capability for the European XFEL", Nucl. Instr. Meth A, 624, 509-519, 2010.

[11] G. Weidenspointner, et al., "Strategy for calibrating the non-linear gain of the DSSC detector for the European XFEL", IEEE NSS\&MIC Proceedings, Valencia, Spain, 2011.

[12] J. D. Hunter, "Matplotlib: A 2D graphics environment", Computing In Science \& Eng., 9, 90-95, 2007. 